\title{
Méthodes comparées de dépistage de Fasciola gigantica chez les bovins dans un abattoir de l'Ouest Cameroun
}

\author{
Alain Kouam Simo ${ }^{1 *}$ Marie-Madeleine Tetda ${ }^{1}$ \\ Mohamed Gharbi ${ }^{2}$ Philippe Dorchies ${ }^{3}$
}

\section{Mots-clés}

Bovin, Fasciola gigantica, abattoir, bile, diagnostic, Cameroun

\footnotetext{
Submitted: 9 September 2019

Accepted: 14 August 2020

Published: 25 January 2021

DOI: $10.19182 /$ remvt.36311
}

\section{Résumé}

Une enquête a été réalisée sur 330 bovins à I'abattoir de Bangangté (région Ouest, Cameroun) pour estimer la prévalence de la fasciolose bovine. Trois méthodes de dépistage ont été comparées : a) l'inspection vétérinaire post mortem, suivie de la dissection des principaux canaux biliaires destinée à mettre en évidence les adultes de Fasciola gigantica ; b) la coproscopie, utilisant la technique de sédimentation; et c) la recherche des œufs dans la bile. Cette dernière méthode, considérée comme la technique de référence, a permis d'identifier 110 bovins (prévalence de $33 \%$ infestés. Des douves adultes ont été retrouvées dans le foie de 24 d'entre eux (prévalence de $7 \%$ ). Quant à la coproscopie, elle était positive chez 20 animaux (prévalence de $6 \%$ ). Tous les bovins positifs à la coproscopie ou lors de l'examen du foie avaient des œufs de F. gigantica dans les échantillons de bile. En revanche, parmi les 306 bovins chez lesquels aucune douve adulte n'a été retrouvée dans le foie, 10 avaient des œufs de $F$. gigantica dans leurs fèces et 86 éliminaient des œufs dans la bile. Comparées à la technique de référence, les sensibilités de la coproscopie et de la recherche des douves adultes dans les foies ont été respectivement de $18 \%$ (20 coproscopies vs 110 animaux avec œufs dans la bile) et de $22 \%$ (24 foies positifs vs 110). Ces résultats montrent que la fasciolose bovine est fréquente chez les animaux abattus à Bangangté, mais que l'examen du foie ou la coproscopie ne permettent pas d'identifier tous les animaux infestés.

- Comment citer cet article : Kouam Simo A., Tetda M.-M., Gharbi M., Dorchies P., 2020. Comparative methods for the detection of Fasciola gigantica in cattle in a slaughterhouse of West Cameroon. Rev. Elev. Med. Vet. Pays Trop., 73 (4): 273-276, doi: 10.19182/remvt.36311

\section{INTRODUCTION}

Le cheptel bovin du Cameroun, estimé à 7,4 millions de têtes, est constitué principalement de zébus (Bos indicus), mais comprend aussi des taurins (Bos taurus) (INSC, 2017). Cet élevage bovin rencontre diverses contraintes liées à l'environnement, aux conditions climatiques, aux difficultés de transport vers les lieux de consommation et aux maladies. Au Cameroun, les bovins sont affectés par différents

1. Université des Montagnes, Faculté des sciences de la santé, BP 208, Bangangté, Cameroun.

2. Laboratoire de parasitologie, Univ. Manouba, Ecole nationale de médecine vétérinaire de Sidi Thabet, 2002 Sidi Thabet, Tunisie.

3. Ecole nationale vétérinaire de Toulouse, 31076 Toulouse, France.

* Auteur pour la correspondance

Email : alkouam2005@yahoo.fr parasites parmi lesquels les protozoaires (notamment les trypanosomes et les coccidies), les strongles, les cestodes et les trématodes (paramphistomes et douves). Fasciola gigantica est la seule douve présente au Cameroun. Elle est à l'origine d'importantes pertes financières (retard de croissance, infertilité relative et baisse de la production laitière) liées aux lésions hépatiques qu'elle provoque, aux troubles métaboliques induits et à l'immunodéficience observée consécutivement à la prédominance des réponses à l'infestation de type Th2 par rapport aux Th1 (O’Neill et al., 2000).

Les pertes de production dues à ce parasite sont grandes. Des résultats obtenus par Loyacano et al. (2002) en Louisiane montrent ainsi que les vaches traitées contre l'infestation par la douve ont un gain de poids de $6 \%$ plus élevé que celles qui ne sont pas traitées. Selon Schweizer et al. (2005) et Mazeri et al. (2017), la fasciolose entraînerait une réduction de la croissance (un animal infesté mettrait en moyenne 10 jours de plus pour atteindre le même poids d'abattage qu'un animal sain), une baisse de la production laitière de $10 \%$ et une 
moindre capacité de travail (augmentation du temps nécessaire pour le labour d'une parcelle suite à la fatigue et à l'anémie qu'entraîne l'infestation). Une étude réalisée au Nigeria montre une prévalence annuelle de $2,5 \%$. Sur une population bovine de 10 millions d'animaux, dont $10 \%$ sont abattus pour la consommation chaque année, les mortalités annuelles sont estimées à $1 \%$ et la saisie des foies à $7 \%$ (Ogunrinade et al., 1980).

Malgré ses effets néfastes sur la santé animale et les pertes économiques engendrées, peu d'informations concernant la fasciolose bovine sont disponibles dans la région Ouest. C'est la raison pour laquelle une enquête a été réalisée à l'abattoir de Bangangté. Il s'agissait en particulier de comparer trois méthodes de dépistage parasitologique : inspection sanitaire, examen coproscopique, et recherche des œufs dans la bile, cette dernière technique étant considérée comme la technique de référence (Rapsch et al., 2006).

\section{MATERIEL ET METHODES}

\section{Région d'étude}

L'étude a été réalisée de mars à août 2017 à l'abattoir municipal de Bangangté dans le département du Ndé (région de l'Ouest, Cameroun). Ce département a une superficie de 923 kilomètres carrés et une population de 100000 habitants. Le climat est de type tropical, la température moyenne mensuelle est comprise entre $14^{\circ} \mathrm{C}$ et $28^{\circ} \mathrm{C}$, et les précipitations annuelles moyennes varient entre 1400 et 2500 millimètres (Anonyme, 2019).

\section{Matériel}

Durant la période d'étude, tous les bovins abattus à l'abattoir de Bangangté ont été examinés. Après abattage, $50 \mathrm{~g}$ de fèces et la totalité de la bile contenue dans la vésicule biliaire ont été collectés et placés dans des boîtes de prélèvements identifiés. Tous les foies ont été examinés par le vétérinaire inspecteur en charge de l'abattoir. Les lésions hépatiques et la présence de douves vivantes ou calcifiées ont été recherchées par deux ou trois sections réglementaires de la face viscérale du foie. L'origine des zébus n'était pas toujours connue. Les animaux provenaient souvent du département de Noun (communes de Founbam et de Foumbot), également dans l'Ouest. De plus, certains animaux passaient par plusieurs intermédiaires qui ne communiquaient pas souvent l'origine des animaux.

\section{Recherche des oufs dans la bile}

Au laboratoire, la bile a été placée dans un verre à pied. Après 15 min de sédimentation, le sédiment a été aspiré avec une pipette puis déposé dans une boîte de Petri sur une hauteur ne dépassant pas $1 \mathrm{~mm}$. Afin de faciliter l'observation des œufs de douves, la solution a été colorée avec quelques gouttes de bleu de méthylène. La recherche des œufs de F. gigantica a été faite sous loupe binoculaire (grossissement x 100).

\section{Coproscopie}

La méthode de sédimentation a été utilisée pour estimer la prévalence d'infestation par $F$. gigantica. Pour ce faire, $5 \mathrm{~g}$ de fèces ont été pesés et mélangés avec $70 \mathrm{ml}$ d'eau de robinet. Le mélange a été placé dans un mortier, homogénéisé avec un pilon, puis filtré et sédimenté pendant $1 \mathrm{~h}$. Une pipette a été utilisée pour prélever doucement le sédiment. La suspension a été colorée avec quelques gouttes de bleu de méthylène et observée sous loupe binoculaire (x 100).

\section{Analyses statistiques}

Les prévalences d'infestation ont été comparées avec le test du khi carré au risque de 5 \% (Schwartz, 1993). La sensibilité a été évaluée par comparaison avec l'examen de la bile, considéré comme la technique de référence.

\section{RESULTATS ET DISCUSSION}

L'objectif a été d'estimer la prévalence de $F$. gigantica parmi les bovins abattus à Bangangté. L'examen post mortem a révélé que $8 \%$ (26/330) des foies présentaient des lésions de fasciolose (cholangite, hépatite parenchymateuse). Des douves adultes ont été retrouvées dans les canaux biliaires de 7,3\% (24/330) des animaux. Le nombre de $F$. gigantica trouvées a varié de 3 à 11 douves chez 10 des animaux infestés et a été supérieur à 12 douves chez les 14 autres. Des œufs de F. gigantica ont été identifiés dans la bile de 110 des 330 bovins examinés (33\%) et dans les fèces de 20 d'entre eux $(6,1 \%)(\mathrm{p}=0,0001)$. L'inspection des foies et la coproscopie n'ont pas permis de détecter tous les animaux identifiés comme infestés par examen de la bile.

Plusieurs méthodes peuvent être utilisées pour estimer la prévalence de la grande douve mais leurs valeurs intrinsèques sont variables. Rapsch et al. (2006) ont comparé ces diverses méthodes de diagnostic sur 1331 bovins pour lesquels la prévalence de $F$. hepatica a été estimée à $18 \%$ : la coproscopie, un test Elisa du commerce, la recherche des œufs dans la bile et le résultat de l'inspection sanitaire. C'est l'examen de la bile qui avait la meilleure sensibilité (93\%).

Lors de l'étude présentée ici, le test Elisa n'a pas été mis en œuvre par manque de moyens financiers. Ceci ne peut toutefois avoir influencé profondément les résultats car la sensibilité des tests est un peu moindre que celle de la recherche d'œufs dans la bile (Rapsch et al., 2006). L'Elisa est un indicateur de la présence ou de la survenue antérieure d'un épisode d'infestation parasitaire. Il est toutefois possible aussi que la séropositivité témoigne d'un contact avec le parasite durant les mois qui précèdent mais que, à cause d'un nombre insuffisant de métacercaires (le stade parasitaire infestant) ingérées par les bovins, ces contacts n'aboutissent pas à une infestation, c'est-à-dire au développement de douves adultes dans les canaux biliaires. Il s'agit alors de faux positifs. En effet, compte tenu de la faible réceptivité des bovins, moins de $10 \%$ des métacercaires achèvent leur évolution chez cette espèce alors que ce pourcentage est plus élevé (30-50 \%) chez les ovins, espèce la plus réceptive (Mekroud et al. 2004). Malgré cela, la recherche des anticorps spécifiques, aussi bien dans le sang que dans le lait, est considérée comme un outil sensible et spécifique pour le dépistage de la grande douve (Chauvin et al., 1997).

La coproscopie est spécifique mais peu sensible pour le dépistage des cas de fasciolose à $F$. gigantica. L'examen d'un échantillon de $10 \mathrm{~g}$ de fèces a toutefois une sensibilité de plus de $60 \%$, mais une telle quantité de matières fécales n'est jamais examinée en routine car le temps nécessaire pour réaliser les coproscopies et le petit nombre d'œufs mis en évidence sont démotivants pour le manipulateur. En augmentant le poids de l'échantillon examiné, la sensibilité est améliorée de manière significative. Charlier et al. (2008) ont ainsi constaté un niveau de sensibilité variable selon la quantité de matières fécales examinée : pour $4 \mathrm{~g}$ la sensibilité est de $43 \%$ mais elle atteint $64 \%$ pour $10 \mathrm{~g}$. Ces résultats concordent avec ceux de Rapsch et al. (2006).

Les conditions de l'enquête n'ont pas permis d'augmenter la taille des échantillons de matières fécales examinés. La prévalence estimée avec la coproscopie $(6 \%)$ était bien inférieure à celle estimée par examen de la bile (33\%); la sensibilité de la coproscopie était donc de $18 \%$ par rapport à l'examen de la bile. Elle était cependant comparable à celles rapportées par Youssao et Assogba (2002) dans la vallée du fleuve Niger (prévalence de $11 \%$ ), et par Mingoas et al. (2006) à Ngaoundéré au Nord Cameroun (prévalence de $8 \%$ ).

La recherche des parasites dans le foie à l'abattoir était un peu plus sensible que la coproscopie (prévalence de $7 \%$ et sensibilité de $22 \%$ ) 
et était très spécifique. Les réglementations européennes 854/2004 et 1244/2007 ne recommandent qu'une inspection visuelle des viandes, c'est-à-dire sans les techniques de routine obligatoires de palpation et d'incision des carcasses ou des viscères mises en œuvre dans la présente étude. Dans ces conditions, un certain nombre de cas de fasciolose échapperait aux inspecteurs mais ce risque serait « négligeable à très faible » (Hill et al., 2014). Il faut cependant souligner que les lésions macroscopiques des canaux biliaires ainsi que la cirrhose hépatique sont moins importantes chez le zébu que chez les bovins européens infestés par F. hepatica (Yilma et Mesfin, 2000).

Les résultats montrent l'insuffisante sensibilité de l'inspection sanitaire et l'intérêt de la recherche des œufs dans la bile. Sur le plan pratique, la mise en œuvre de cette technique est très simple et permet d'obtenir un résultat fiable même lorsque les conditions du travail en laboratoire sont difficiles.

\section{CONCLUSION}

La présente étude avait pour but d'estimer la prévalence de $F$. gigantica à l'abattoir de Bangangté dans l'Ouest Cameroun et de comparer trois méthodes de diagnostic expérimental. Elle a montré, par examen de la bile, la présence de la grande douve du foie chez un tiers des bovins tout-venant. Ceci doit motiver les différents acteurs à intensifier le dépistage et la lutte contre la fasciolose chez les bovins. L'inspecteur sanitaire devrait renforcer les moyens d'analyse du cinquième quartier (les abats). Enfin, les éleveurs doivent être sensibilisés sur les pertes économiques provoquées par la présence de ces parasites chez leurs animaux.

\section{Remerciements}

Les auteurs remercient tous les bouchers et les responsables de l'abattoir qui les ont autorisés à prélever les foies.

\section{Déclaration des contributions des auteurs}

PD et AKS ont conçu et planifié l'étude ; MMT et AKS ont recueilli les données et rédigé la première version du manuscrit ; PD et MG ont révisé le manuscrit.

\section{Conflits d'intérêts}

Les auteurs déclarent que l'étude a été réalisée sans conflit d’intérêts.

\section{REFERENCES}

Anon., 2019. Commune de Bangangté. Milieu biophysique : le climat. www. communedebangangte.net/non-categorise/milieu-biophysique-le-climat/ (consulté le 16/08/2019)

Charlier J., De Meulemeester L., Claerebout E., Williams D., Vercruysse J., 2008. Qualitative and quantitative evaluation of coprological and serological techniques for the diagnosis of fasciolosis in cattle. Vet. Parasitol., 153 (1-2): 44-51, doi: 10.1016/j.vetpar.2008.01.035

Chauvin A., Moreau E., Boulard C., 1997. Diagnostic de la fasciolose bovine par sérologie de mélange. Interprétation en conditions de terrain. Vet. Res., 28 (1): $37-43$

Hill A.A., Horigan V., Clarke K.A., Dewé T.C.M., Stärk K.D.C., O’Brien S., Buncic S., 2014. A qualitative risk assessment for visual-only post-mortem meat inspection of cattle, sheep, goats and farmed/wild deer. Food Control, 38: 96-103, doi: 10.1016/j.foodcont.2013.10.002

INSC (Institut National de la Statistique du Cameroun), 2017. Annuaire Statistique du Cameroun. Chapitre 15 : Élevage et Pêche. INSC, Yaoundé, Cameroun, http://onsp.minsante.cm/sites/default/files/publications/237/Annuaire\%20Statistique\%202017.pdf (consulté le 20/08/2020))

Loyacano A.F., Williams J.C., Gurie J., DeRosa A.A., 2002. Effect of gastrointestinal nematode and liver fluke infections on weight gain and reproductive performance of beef heifers. Vet. Parasitol., 107 (3): 227-234, doi : 10.1016/S0304-4017(02)00130-9

Mazeri S., Rydevik G., Handel I., Bronsvoort B.M.D., Sargison, N., 2017. Estimation of the impact of Fasciola hepatica infection on time taken for UK beef cattle to reach slaughter weight. Sci. Rep., 7(1): 7319, doi: 10.1038/ s41598-017-07396-1

Mekroud A., Benakhla A., Vignoles P., Rondelaud D., Dreyfuss G., 2004. Preliminary studies on the prevalences of natural fasciolosis in cattle, sheep, and the host snail (Galba truncatula) in north-eastern Algeria. Parasitol. Res., 92 (6): 502-505, doi: 10.1007/s00436-004-1072-1

Mingoas K.J.P., Gambo H., Ottou J.F.B., Ebangi A.L., Domwa M., 2006. Structure des troupeaux et conduite de l'Elevage bovin périurbain de Ngaoundéré au Cameroun. Bull. Anim. Health Prod. Africa, 54 (1): 53-65, doi: 10.4314/bahpa.v54i1.32730

O’Neill S.M., Brady M.T., Callanan J.J., Mulcahy G., Joyce P., Mills K.H.G. Dalton J.P., 2000. Fasciola hepatica infection downregulates Th1 responses in mice. Parasite Immunol., 22 (3): 147-155, doi: 10.1046/j.13653024.2000.00290.x

Ogunrinade A., Ogunrinade B.I., 1980. Economic importance of bovine fascioliasis in Nigeria. Trop. Anim. Health Prod., 12 (3): 155-160, doi: 10.1007/ bf02242647

Rapsch C., Schweizer G., Grimm F., Kohler L., Bauer C., Deplazes P., Braun U., et al., 2006. Estimating the true prevalence of Fasciola hepatica in cattle slaughtered in Switzerland in the absence of absolute diagnostic test. Int. J. Parasitol., 36 (10-11): 1153-1158, doi: 10.1016/j.ijpara.2006.06.001

Schwartz D., 1993. Méthodes statistiques à l'usage des médecins et des biologistes, 4e Ed. Flammarion, Paris, France, 315 p.

Schweizer G., Braun U., Deplazes P., Torgerson P.R., 2005. Estimating the financial losses due to bovine fasciolosis in Switzerland. Vet. Rec., 157 (7): 188-193, doi: 10.1136/vr.157.7.188

Yilma J.M., Mesfin Y., 2000. Dry season bovine fasciolosis in Northwestern part of Ethiopia. Rev. Méd. Vét., 151 (6): 493-500

Youssao A.K.I., Assogba M.N., 2002. Prevalence of cattle fasciolosis in the Niger River valley of Benin. Rev. Elev. Med. Vet. Pays Trop., 55 (2): 105108, doi: 10.19182/remvt.9830 


\section{Summary}

Kouam Simo A., Tetda M.-M., Gharbi M., Dorchies P. Comparative methods for the detection of Fasciola gigantica in cattle in a slaughterhouse of West Cameroon

A survey was conducted on 330 cattle at Bangangté's slaughterhouse (West Region, Cameroon) to estimate the prevalence of bovine fasciolosis. Three screening methods were compared: i) postmortem veterinary inspection, followed by dissection of the main bile ducts to detect adults of Fasciola gigantica; ii) coproscopy with the sedimentation technique; and iii) the search for eggs in the bile. The latter method, considered as the gold standard, identified 110 infected cattle (33\% prevalence). Adult flukes were found in the liver of 24 of them (7\% prevalence). The coproscopy was positive in 20 animals $(6 \%$ prevalence). All the cattle that were found positive by the coproscopy or liver examination had $F$. gigantica eggs in their bile samples. In contrast, of the 306 cattle in which no adult fluke was found in their livers, 10 had $F$. gigantica eggs in their feces and 86 eliminated eggs in the bile. Compared to the gold standard, the sensitivity of coproscopy and the search for adult flukes in livers were 18\% (20 coproscopies vs 110 animals with eggs in bile) and $22 \%$ (24 positive livers vs 110 ), respectively. These results show that bovine fasciolosis is common in animals slaughtered in Bangangté, but that liver examination or coproscopy cannot identify all infected animals.

Keywords: cattle, Fasciola gigantica, abattoirs, bile, diagnosis, Cameroon

\section{Resumen}

Kouam Simo A., Tetda M.-M., Gharbi M., Dorchies P. Métodos comparativos de detección de Fasciola gigantica en los bovinos de un matadero en Oeste Camerún

Se realizó una investigación post mortem en 330 bovinos en el matadero de Bangangté (región Oeste, Camerún), con el fin de estimar la prevalencia de la faciolosis bovina. Se compararon tres métodos de detección: a) la inspección veterinaria post mortem, seguida de la disección de los principales canales biliares destinada a poner en evidencia los adultos de Fasciola gigantica; b) la coproscopía, utilizando la técnica de sedimentación; y c) la búsqueda de huevos en la bilis. Este último método, considerado como la técnica de referencia, permitió identificar 110 bovinos (prevalencia 33\%) infestados. Trematodos adultos fueron encontrados en el hígado de 24 bovinos (prevalencia de 7\%). En cuanto a la coproscopía, fue positiva en 20 animales (prevalencia de 6\%). Todos los bovinos positivos por coproscopía o durante el examen del hígado presentaron huevos de $F$. gigantica en las muestras de bilis. Por otro lado, entre los 306 bovinos en los cuales no se encontró ningún trematodo adulto en el hígado, 10 tenían huevos de F. gigantica en las heces y 86 eliminaron huevos en la bilis. Comparados a la técnica de referencia, las sensibilidades de la coproscopía y de la búsqueda de trematodos adultos en el hígado fueron respectivamente de 18\% (20 coproscopías vs 110 animales con huevos en la bilis) y de $22 \%$ (24 hígados positivos vs 110). Estos resultados muestran que la faciolosis bovina es frecuente en los animales de matadero en Bangagté, pero que el examen del hígado o la coproscopía no permiten identificar todos los animales infestados.

Palabras clave: ganado bovino, Fasciola gigantica, mataderos, bilis, diagnóstico, Camerún 